\title{
Sobre la posible secuencia uvas et ficus en un fragmento de cerámica de Gayo Valerio Verdulo hallado en el yacimiento de las Eras de San Martín de Alfaro*
}

\author{
About the probable sequence uvas et ficus in a piece of Gaius \\ Valerius Verdullus' pottery founded in the Eras de San Martín of \\ Alfaro (La Rioja, Spain) \\ Marc MAYER I OLIVÉ
}

\begin{abstract}
RESUMEN
ABSTRACT

Un fragmento de cerámica atribuible a Gayo Valerio Verdulo contiene un texto fragmentario que presenta una probable reminiscencia de Lucilio y concomitancias con otros autores latinos, como Horacio y Marcial.

PALABRAS CLAVE

Gayo Valerio Verdulo, Horacio, Marcial, Literatura Latina, Epigrafía.
\end{abstract}

Recibido el 25 de abril de 2012. Aceptado el 5 de agosto de 2012

Continuando con el estudio de las posibles referencias intertextuales en los letreros contenidos en la cerámica producida por Gaius Valerius Verdullus, podemos en esta ocasión presentar un nuevo posible caso de reminiscencia de singular trascendencia ${ }^{1}$. Un pasaje de Lucilio podría estar relacionado con la expresión [- - -

*Este trabajo se inscribe en el proyecto de investigación FFI2011-25113 y en el Grup de Recerca consolidat LITTERA (2009SGR105). Marc Mayer i Olivé, Catedrático de Filología Latina, Universidad de Barcelona, mayer@ub.edu.

${ }^{1}$ Cf. por ejemplo: GONZÁLEZ BLANCO GARCÍA, Elena, «La primera obra de teatro conocida en La Rioja, un mimo del siglo primero representado en Calahorra: a propósito del vaso erótico del alfarero Verdullo hallado en La Maja», Kalakorikos, 7, (2002), pp. 203-208; MAYER, Marc, «Los Xenia de Marcial clave de interpretación de un vaso figurado del Alfar de La Maja (Calahorra, La Rioja)», en ISO ECHEGO- 
]AS.ET.FICVS, presente en un fragmento de la cerámica de Verdullus², que se puede reconstruir, como creemos, de acuerdo con la iconografía: [- - VV]AS.ET.FICVS (fig. 1) ${ }^{3}$.

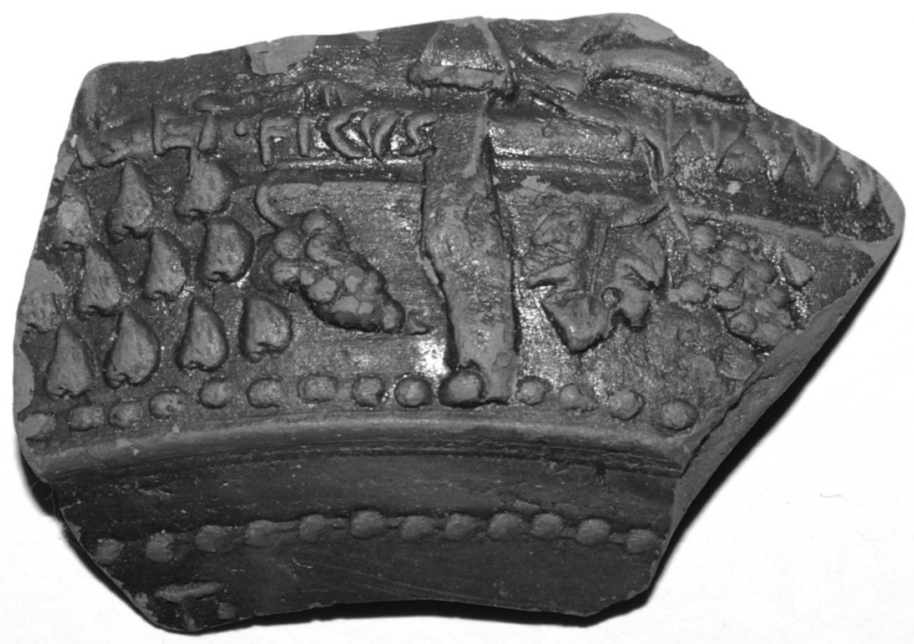

Fig. 1. Fragmento de pared del vaso de Gayo Valerio Verdulo con la leyenda:]AS $\bullet$ ET• FICVS, encontrado en Alfaro, La Rioja. Fotografía cedida por José Luís Cinca.

YEN, José Javier, ed., Hominem pagina nostra sapit. Marcial, 1900 años después. Estudios XIX centenario de la muerte de Marco Valerio Marcial. Zaragoza 2004, pp. 115-127, con el elenco bibliográfico de lo publicado hasta el momento de este yacimiento; VELAZA, Javier, «El «vaso del zódiaco» de Gayo Valerio Verdulo: problemas de reconstrucción y de interpretación», en Espacios, usos y formas de la epigrafía hispana en épocas antigua y tardoantigua. Homenaje al Dr. Armin U. Stylow, (Anejos de AEspA, XLVIII). Mérida 2009, pp. 363-373, con la bibliografía anterior; MAYER, Marc, «El mito de Hipólito según la versión de la Fedra de Séneca, representado en un vaso de cerámica producida en La Maja (Calahorra, La Rioja)», Kalakorikos, 15, (2010), pp.97-108; ID., «Notas preliminares y conjeturas sobre el posible significado de algunos de los letreros presentes en los vasos de Gayo Valerio Verdulo del alfar de La Maja», Kalakorikos, 16, (2011), pp. 123-137, esp. pp. 133-136. La iconografía en relación con los textos ha sido tratada en sucesivas contribuciones de BARATTA, Giulia, «Un primo approccio all'iconografia del mito di Ippolito sulla ceramica di Gaius Valerius Verdullus», Kalakorikos, 15, (2010), pp. 109-120; EAD., «Sull'iconografia di alcuni frammenti della produzione cerámica di Gaius Valerius Verdullus: un nuovo soggetto iconográfico ispirato forse al mondo funerario", Kalakorikos, 16, (2011), pp. 139-160. En un sentido semejante limitado a la serie denominada «erótica, cf. Stramaglia, Antonio, «ll fumetto e le sue potenzialità mediatiche nel mondo greco-latino» en FERNÁNDEZ DELGADO, José Antonio, PORDOMINGO, Francisca, STRAMAGLIA, Antonio, eds., Escuela y literatura en la Grecia Antigua. Actas del Simposio Internacional, Universidad de Salamanca 17-19 Noviembre de 2004. Cassino 2007, pp. 577-643, 24 láms., esp. pp. 604-610 y lám. 17. mento.

${ }_{2}^{2}$ Queremos agradecer al amigo José Luís Cinca Martínez el envio de la fotografía de este frag-

${ }^{3}$ El fragmento fue publicado por primera vez por MARTÍNEZ TORREClLLA, José Manuel, «Un fragmento de vaso decorado de paredes finas de G. Val. Verdullus procedente de Graccurris", Kalakorikos, 12, (2007), pp. 271-273, con una cuidada descripción y la indicación de las circunstancias arquelógicas del hallazgo, proponiendo la lectura: [...]IS.ET.FICVS. Remitimos en consecuencia a este trabajo y el que mecionamos a continuación para la entidad material de este fragmento; cf. BARATTA, Giulia, «Sull'iconografia di alcuni frammenti...», núm. 10, pp.157-158. 
El fragmento de Lucilio en sus Sátiras (frag. 1173 Marx; 1111 Krenkel; H 58 Charpin) reza como sigue: <-uu -uu ->fici comeduntur et uvae 4 . No entraremos en los problemas gramaticales de ficus bien recogidos por F. Charpin en su edición ${ }^{5}$, pero hay que destacar que Carisio por dos veces repite este hemistiquio de Lucilio ( GLK 1, 95,22 y 1, 129, 2), la primera vez después de una cuestión gramatical y la segunda después de citar a Varrón, concretamente el libro primero del De scaenicis originibus, donde se halla una referencia al ficus Ruminalis.

Con buen criterio F. Charpin señala, en la correspondiente nota complementaria $^{6}$, que las uvas y los higos eran el postre más simple y corriente para una comida sencilla y aduce como ilustración un pasaje de Horacio, sat. 2, 2, 118-121, y propone relacionar, además, este fragmento con otro del propio Lucilio donde habla de un victus honestus (frag. 1288 Marx; 1305 Krenkel; H 52 Charpin) ${ }^{7}$. Lo mismo hace W. Krenkel a propósito del fragmento que nos ocupa ${ }^{8}$.

La tradición posible de la leyenda presente en el vaso de Verdullus resulta, pues, clara y puede remontarse, como tantos otros aspectos de las expresiones literarias romanas, al propio Lucilio. No entraremos aquí por su obviedad en la frecuente presencia en paralelo de la secuencia uvae y fici o ficus ${ }^{9}$, pero sí debemos pensar en integrar la expresión de la que forman parte en el contexto iconográfico del modelo de vaso de La Maja, al que pudo pertenecer.

En un trabajo reciente $\mathrm{G}$. Baratta ha propuesto que el fragmento formaría quizás parte del mismo vaso que contiene la leyenda cave fossa $(m)$ y fossa $(m)$ cave, que nosotros también comentamos en un trabajo anterior (figs. 2 y 3$)^{10}$, haciéndonos eco de la propuesta de integración del fragmento que ahora comentamos, pero sin tratar de reconstruir el posible significado que permite quizás entrever la parte del texto conservada. El hecho de que se pueda unir a los demás fragmentos como propone esta estudiosa nos permite atribuir la producción al alfar localizado en La Maja, Pradejón, La Rioja. El vaso representaría, en principio posiblemente un hortus funerario ${ }^{11}$, en forma de recinto cerrado por una pared o ba-

\footnotetext{
${ }^{4}$ Véase KRENKEL, Werner, ed., Lucilius Satiren, vol. II. Leiden 1970, pp. 594-595, que recontruye: - uu -uu fíci inquit cómedúntur et úvae, un hexámetro, y lo pone en relación con el fragmento 1112 de su edición: ádsiduas ficós uu -uu -uu -uu, (frag. 1101 Marx); atribuyéndolos a los libros I-XXV o XXVIII$\mathrm{XXX}$.

${ }^{5}$ CHARPIN, François, ed., Lucilius, Satires, tome III, Livres XXIX, XXX et fragments divers. Paris 1991, pp. 117-118.

${ }^{6}$ CHARPIN, François, ed., Lucilius..., III, p. 273.

${ }^{7}$ CHARPIN, François, ed., Lucilius..., III, p. 115.

${ }^{8}$ KRENKEL, Werner, ed., Lucilius Satiren, II, p. 595: «vgl. Hor. sat. 2, 2 121: Tum pensilis uva secundas / Et nux ornabat mensas cum duplice ficu». Sobre la relación estrecha entre Lucilio y Horacio véase el ya clásico estudio de FISKE, George Converse, Lucilius and Horace. A Study in the Classical Theory of Imitation. Madison 1920, (reimpr. Westford, Conn. 1971), esp. pp. 369-424, para el libro segundo de las Sátiras de Horacio, y p. 385, para el pasaje que tratamos, que considera claramente derivado de Lucilio.

${ }^{9}$ ThIL, VI, s.v. ficus, cols. 650-654, (Vollmer), esp col. 652, la nux, o mejor las nuces, es el tercer elemento en frecuencia que se combina en paralelo con uvae y fici.

10 BARATTA, Giulia, «Sull'iconografia di alcuni frammenti...», pp. 139-160, esp.p. 144; MAYER, Marc, «Notas preliminares...», ibidem, pp. 123-137, esp. p. 132.

${ }_{11}$ Para los jardines funerarios, cf. ToYNBEE, Jocelyn Mary Catherine, Death and Burial in the Roman World. Baltimore, London 1996 (1 ${ }^{\text {a }}$ ed. London 1971), pp. 94-100, 299-301; TIRELLI, Margherita, «Hor-
} 
laustrada, con una abundante vegetación, elemento frecuente como resulta de los ejemplos arqueológicamente documentados, pero por el momento único en su representación como decoración de un vaso cerámico.

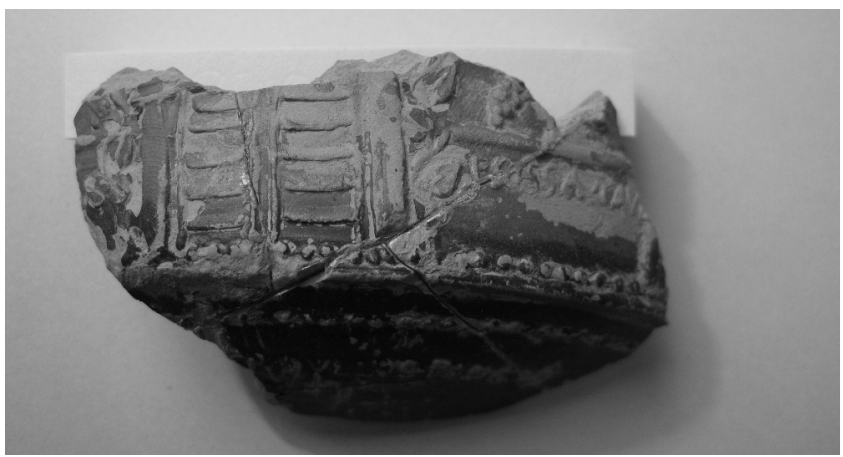

Fig. 2. Fragmento de vaso de Verdulo hallado en el yacimiento de La Maja Pradejón, La Rioja, con la leyenda FOSSA CAVE. Museo de la Rioja, núm. de inventario LMJ/K27/10047.6.

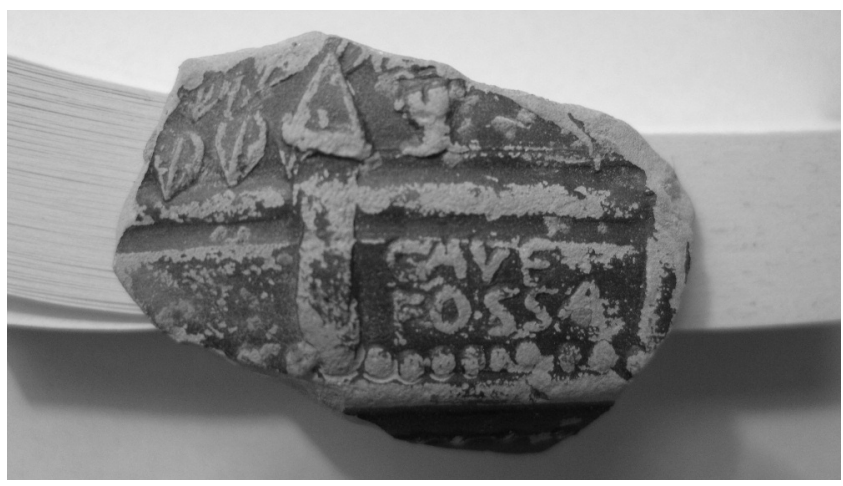

Fig. 3. Fragmento de vaso de Verdulo con la leyenda CAVE / FOSSA, hallado en 1996 en La Maja, Pradejón, La Rioja. Museo de la Rioja, núm. de inventario LMJ/LL25/12.008.

ti cum aedificiis sepulturis adiuncti.I monumenti funerari nelle necropoli di Altinum», en MIRABELLA ROBERTI, Mario, ed., Monumenti sepulcrali Romani in Aquileia, (AA, XLIX). Trieste 1997, pp. 169-210; ORTALLI, Jacopo, «La via dei sepolcri di Sarsina. Aspetti funzionali, formali e sociali», en VON HESBERG, Henner, ZANKER, Paul, eds., Römische Grabstrassen. Selbstdarstellung-Status-Standard, Kolloquium in München vom 28. bis 30. Oktober 1985, (Bayerische Akad, der Wissenschaften. Phil.-hist. Klasse, Abh. N. F. Heft 96.). München 1987, pp. 155-182; VERZÁR-BASS, Monica, «A proposito dei mausolei negli horti e nelle ville», en CIMA, Maddalena, LA ROCCA, Eugenio, eds., Horti romani. Atti del congresso internazionale, Roma, 4-6 maggio 1995, Roma 1998, pp. 401-424; FRASS, Monika, Antike römische Gärten. Soziale und wirtschaftliche Funktionen der Horti Romani, (Gräzer Beiträge, Supplementband X). Horn/Wien 2006; REBENICH, Stefan, «Garten, Gräber und Gedächtnis. Villenkultur und Bestattungspraxis in der römischen Kaiserzeit», en BÖRM, Henning, EHRHARDT, Norbert, WIESEHÖFER, Josef, eds., Monumentum et instrumentum inscriptum. Beschriftete Objekte aus Kaiserzeit und Spätantike als historiker Zeugnisse. Festschrift für Peter Weiss zum 65. Geburtstag. Stuttgart 2008, pp. 187-201 
El texto que se puede reconstruir, uvjas et ficus, parece dar una secuencia métrica - - - -, que parece ser la parte final del texto a juzgar por su situación en el fragmento conservado. Consecuentemente sería posible conjeturar, si queremos creer en un texto en verso, que se trataría de un final de ocho unidades de duración, que naturalmente se puede entender como dos espondeos, lo que evidentemente podrían corresponder al final de un hexámetro dactílico, aunque la substitución de dáctilo por espondeo no es frecuente en el quinto pie ${ }^{12}$, como sería este el caso si pensamos en un final de verso, aunque, evidentemente, resulta posible. Podría también, si queremos continuar pensando en un texto métrico, tratarse, menos probablemente, del final de un septenario yámbico. De todos modos, aunque presente indudables reminiscencias poéticas, el texto no tiene obligatoriamente que pertenecer a esta categoría.

La iconografía presente en el fragmento no puede ser más explícita, se integre éste o no en el modelo de vaso antes mencionado. Vemos en ella uvas e higos y a partir de ello, y teniendo presente el posible componente o raíz lucilianos de la expresión, podemos intentar reconstruir el ambiente literario en que se pudo integrar el texto de Gayo Valerio Verdulo. Habida cuenta de los antecedentes, no nos cabe duda que quizás volvamos a estar ante un elemento literario vinculado a los Saturnalia, y hemos de recordar aquí de nuevo, como elemento probatorio, el vaso con representación de xenia, que halla su correspondencia en Marcial ${ }^{13}$.

Es precisamente de nuevo en Marcial y concretamente en el libro décimotercero, los Xenia, donde podemos hallar un pasaje ${ }^{14}$ que puede resultar iluminador para nuestro texto, en el que el poeta hace hablar a una ficedula, que es ella misma objeto de regalo, y que empareja en su breve definición, con gran sentido irónico, los dos elementos que nos ocupan:

Cum me ficus alat, cum pascar dulcibus uvis,

Cur potius nomen non dedit uva mihi.

El paralelismo resulta evidente y cumple precisamente los parámetros de simplicidad en que se movía el fragmento de Lucilio, que muy probablemente está en el origen de toda esta tradición, aunque la versión de Marcial no esté exenta de un cierto preciosismo. El tono humorístico indudable viene dado por la

12 Cf. por ejemplo, para valorar la poca frecuencia del caso los esquemas recogidos en el trabajo de MARINA SÁEZ, Rosa María, La métrica de los Epigramas de Marcial. Esquemas rítmicos y esquemas verbales. Zaragoza1998.

${ }^{13}$ Cf. para este vaso MAYER, Marc, «Los Xenia de Marcial...», pp. 115-127.

${ }^{14}$ MART. 13, 49, poema que lleva por lema ficedulae. Se trata del famoso pájaro conocido como papahigos, denominado en italiano «beccafico», que es quien toma la palabra en el corto poema. Cf. para la presencia de ficus en Marcial: ESTEFANÍA, Dulce, M. Val. Martialis epigrammaton concordantia, fasc. $4^{\circ} E-F$. Santiago de Compostela 1985, pp. 973-974. 
implícita protesta de la ficedula por el hecho de que su nombre derive de ficus y no de uva cuando se nutre de uno y otro fruto ${ }^{15}$; evidentemente, si buscamos un doble sentido al pasaje del poeta de Bilbilis, no se puede negar que éste también puede estar implícito, e incluso nos atreveríamos a decir que casi explícito, en el mismo ${ }^{16}$.

Sin buscar dobles sentidos, no cabe duda también de que las uvas y los higos son por sí mismos un regalo típico de los Saturnalia, como demuestra el propio Marcial en otro conocido pasaje ${ }^{17}$ :

Omnia missisti mihi Saturnalibus, Vmber, munera, contulerant quae tibi quinque dies:

bis senos triplices et dentiscalpia septem;

his comes accessit spongea, mappa, calix, semodiusque fabae cum vimine Picenarum

et Laletana nigra lagona sapae;

parvaque cum canis venerunt cottana prunis

et Lybicae fici pondere testa gravis.

Vix puto triginta nummorum tota fuisse

munera quae grandes octo tulere Syri.

quanto commodius nullo mihi ferre labore

argenti potuit pondera quinque puer!

Lo mismo sucede en otro poema del mismo Marcial, el cual empieza: Saturnalia divitem Sabellum / fecerunt..., donde recuerda de nuevo los regalos de estas celebraciones y menciona explícitamente: et ficus Lybica gelata testa ${ }^{18}$.

${ }^{15}$ Así la variatio de persona y de verbo en el caso de alat y pascar ha sido bien valorada en los comentarios, cf. por ejemplo el muy reciente de LEARY, T.J., Martial. Book XIII. The Xenia. London 2001, p. 102; y también el ya clásico de FRIEDLAENDER, Ludwig, M. Valerii Martialis epigrammaton libri, vol. II. Leipzig 1886, p. 279, que no hace comentario alguno al respecto.

${ }^{16}$ Véase por ejemplo VORBERG, Gaston, Glossarium eroticum. Roma 1985 (repr. anastática de la edición de Stuttgart 1932), s. v. «ficus», p. 194, donde además remite al uso que hace de la palabra el propio Marcial $(6,53,11)$ y da como sinónimo marisca; cf. también, s.v. «uva», p. 608.

${ }^{17}$ MART. 7, 53, citamos según la edición de LINDSAY, Wallace Martin, M. Val. Martialis epigrammata. Oxford 1903, aunque tenemos también en cuenta la edición teubneriana de SHACKLETON BAILEY, David Roy, M. Valerii Martialis Epigrammata. Stuttgart 1990, p. 229; cf. FRIEDLAENDER, Ludwig, M. Valerii Martialis..., I, p. 501 .

${ }^{18}$ MART. 4, 66, 10, cf. FRIEDLAENDER, Ludwig, M. Valerii Martialis epigrammaton libri, vol. I, p. 360. El poema es particularmente cercano a la descripción de los Xenia. 
El uso de ficus y no de ficos también queda justificado por un nuevo poema de Marcial' ${ }^{19}$ :

Cum dixi ficus, rides quasi barbara verba

Et dici ficos, Laetiliane, iubes.

Dicemus ficus quas scimus in arbore nasci.

Dicemus ficos, Laetiliane, tuos.

De nuevo el doble sentido de ficus es aquí explícito, en un nuevo toque del humorismo, a veces muy directo, de Marcial. En nuestra opinión el criterio de selección de Verdulo se ha movido en los parámetros señalados en estos versos del poeta Marcial ${ }^{20} \mathrm{y}$, una vez más, nos hallamos ante similitudes que hacen pensar en un ambiente cultural común.

Si queremos pensar en el valor obsceno de uvas et ficus, que indudablemente es posible, y por lo demás está bien documentado para ambos términos ${ }^{21}$, podríamos también mencionar, sólo a título de ejemplo que los reúne, el famoso esgrafiado de Baelo, Cádiz, que reza textualmente 22 : hic eg[o] me memin[i qu]ondam futuisse puellam / in cuiiu[s] cunno frig[o]re paene peri / uteritu[- - -] qui[- - -]aes / [- -]a[- - -]qua[- - -] / [cul]us habet f[i]co[s et cunnu]s possidet uvas / [qu]os ambos [refugit] ment[ula] re[s d]ocuit V/ [- - -]OSPNO. Resulta evidente que las uvas et ficus del texto que comentamos pueden enumerar particularidades sexuales o físicas negativas, aunque hemos de notar aquí el uso de ficos en el ejemplo que hemos traído a colación y no de ficus. Lo cual, teniendo además en cuenta el poema de Marcial antes recogido, nos llevaría de nuevo a pensar en el sentido que podríamos denominar agrícola de nuestro texto.

Es interesante observar, en cambio, como el término ficus no se halla presente en Virgilio, el gran pilar de la formación escolar romana, ni siquiera en el encendido elogio de la Saturnia tellus en la Geórgica segunda donde, por el contrario, sí se menciona la uva: sufficiet Baccho vitis, hic fertilis uvae ${ }^{23}$.

${ }^{19}$ MART. 1, 65. 1-4.; cf. CITRONI, Mario, ed., M. Valerii Martialis epigrammaton liber I. Firenze1975, (Biblioteca di Studi Superiori, LXI), pp. 211-214, cuyo texto tomamos, para la discrepancia Caeciliane / Laetiliane ésta última de transmisión indirecta cf. FRIEDLAENDER, Ludwig, M. Valerii Martialis..., I, p. 206. Cf. también SHACKLETON BAILEY, David Roy, M. Valerii Martialis..., p. 66, donde recoge Laetiliane en el texto.

${ }^{20}$ Lo hace también Prisciano en inst. gr. 6, 83, donde recuerda la autoridad del testimonio de Marcial.

${ }^{21}$ Véase nota 11; cf. además CLE 50 C= CIL IV 1820: Chie opto tibi ut<i> refricent se ficus tuae / ut peius ustulentur quam ustulatae sunt, cf. COURTNEY, Edward, Musa lapidaria. A Selection of Latin Verse Inscriptions. Atlanta, Ge. 1995, (Am. Class. Stud., 36), núm 83, pp. 94-95 y 302.

${ }_{22}$ BONNEVILLE, Jean-Noël, DARDAINE, Sylvie, LE ROUX, Patrick, Belo V. L'épigraphie. Les inscriptions romaines de Baelo Claudia. Madrid 1988, (Publications de la Casa de Velazquez. Série Archéologie, X), núm. 105, pp. 95-96 y lám. XL. Cf. MAYER, Marc, «Las inscripciones pintadas en Hispania. Estado de la cuestión», en SolIN, Heikki, SALOMIES, Olli, LIERTZ, Uta-Maria, eds., Acta colloquii epigraphici Latini Helsingiae 3.-6. sept. habiti, Commentationes Humanarum Litterarum. Societas Scientiarum Fennica (Helsinki), 104, 1995, pp. 79-92, esp. p. 83.; cf. además COURTNEY, Edward, Musa lapidaria..., núm. 94 c, pp. $98-99$ y 308, que no publica más que la parte inicial del texto.

${ }^{23}$ VERG. Georg. 2, 191. 
Volviendo de nuevo a nuestro texto y su significado, en el caso que queramos aceptar la vinculación del fragmento cerámico que lo contiene a los fragmentos del vaso con el letrero cave fossa $(m)$ y a la representación de un recinto que parece rodear un hortus, que quizás pudiera, como hemos conjeturado, ser funerario, Juvenal nos proporciona en este caso un pasaje donde ficus aparece en relación con el ambiente funerario 24 :
...tanto maior famae sitis est quam
virtutis. Quis enim virtutem amplectitur ipsam,
praemia si tollas? Patriam tamen obruit olim
gloria paucorum et laudis titulique cupido
haesuri saxis cinerum custodibus, ad quae
discutienda valent sterilis mala robora fici,
quandoquidem data sunt ipsis quoque fata sepulcris.

Las parras y las higueras podrían adornar, cubrir y, a un mismo tiempo, destrozar los sepulcros, lo cual resultaría especialmente significativo para nuestro caso si quisiéramos tomar en cuenta de nuevo la posible significación funeraria, que podría suponerse para el conjunto de fragmentos del que podría formar parte el que aquí tratamos. Recordemos, sin embargo, que toda la hipótesis descansa en la interpretación del término fossa como sepulcro, hecho que no resulta del todo evidente, dada la polisemia del término, cuya significación principal no está vinculada a la designación de una fosa funeraria, aunque documentos más tardíos que la producción cerámica de La Maja vayan perfilando este último significado, así CIL II 1088, nos permite ver cómo este término va cobrando un valor equivalente a tumulus 25 .

Son estas las primeras reflexiones que queríamos proponer a la vista de un texto incompleto y vinculado a un conjunto de fragmentos con los que tiene concomitancias decorativas, aunque no hay puntos de contacto físico entre los fragmentos que permitan integraciones seguras. El resultado de las mismas es de todas maneras bastante evidente: la relación con el ambiente cultural del que surge la obra de Marcial o en el que circula parece un hecho probado, sea por la selección de la forma ficus, sea por la vinculación a los Saturnalia que parece tener una buena parte de la producción de Verdulo, circunstancia que sería especialmente

${ }^{24}$ IVV. 10, 140- 146; citamos según la edición de KNOCHE, Ulrich, D. Iunius luvenalis saturae. München 1950, pp. 96-97; cf. FRIEDLAENDER, Ludwig, D. Junii Juvenalis saturarum libri V, vol I. Leipzig 1895, p. 469, donde no insiste sobre el tema; y COURTNEY, Edward, A Commentary on the Satires of Juvenal. London 1980, p. 468, sin atención a esta cuestión.

${ }^{25}$ CLE 541, CILA Sevilla 305 cf. FERNÁNDEZ MARTÍNEZ, Concepción, Carmina Latina Epigraphica de la Bética Romana. Las primeras piedras de nuestra poesía. Sevilla 2007, SE 1, pp.289-299, (Fernández Martínez, Concepción, Carande Herrero, Rocío), donde en el verso 18 se menciona una fos(s)a $\operatorname{im}[a]$, p. 298. 
adecuada al emparejamiento entre uvas e higos que suponemos, fundadamente, para nuestro texto.

Ir más allá en nuestro razonamiento significaría abandonar hipótesis y conjeturas justificadas para entrar en un terreno solamente especulativo. La coherencia con lo que hasta ahora hemos ido pudiendo deducir sobre Gayo Valerio Verdulo y la cultura de la Calagurris romana y de su zona inmediata no es en modo alguno un resultado que se pueda menospreciar, y resulta un buen elemento de extrapolación a la hora de evaluar lo que pudo ser el nivel y el desarrollo cultural de las provincias hispanas a caballo entre los siglos I y II d.C., concretamente en un territorio que vió nacer en Bilbilis a Marcial y en la propia Calahorra, Calagurris, a Quintiliano, ambiente en el cual a buen seguro Verdulo no fue una excepción. Un ejemplo más de un fenómeno que, sin duda alguna, interesaría de nuevo a quien recordamos en estas páginas, que estudió infatigablemente la sociedad romana hispana. 
